Original Article

\title{
ROLE OF NITRIC OXIDE (NO) IN CAPSAICIN MEDIATED ANTI-PLATELET ACTIVITY IN IN VITRO, IN VIVO, EX-VIVO MODEL OF PLATELET AGGREGATION ASSAY AND ARTERIAL THROMBOSIS IN RAT: POTENTIAL THERAPEUTIC TARGET?
}

\author{
MIHIR K. PATEL ${ }^{1}$, KIRANJ K. CHAUDAGAR ${ }^{1}$, ANITA A. MEHTA ${ }^{1 *}$ \\ Department of Pharmacology, L. M. College of Pharmacy, Navarangpura, Ahmedabad \\ Email: dranitalmcp@gmail.com
}

Received: 06 Feb 2017 Revised and Accepted: 24 Feb 2018

\begin{abstract}
Objective: Although recent advances in the treatment of congestive heart disease, mortality among patients' remains a questionable remark. Therefore, we evaluated the role of capsaicin on in vitro and ex vivo platelet aggregation induced by Adenosine Di-Phosphate (ADP) as well as in in vivo thrombosis models and role of NO, $\mathrm{K}_{\mathrm{ATP}}$ was also identified in the capsaicin-induced anti-platelet animal model as well as in vivo model of arterial thrombosis.
\end{abstract}

Methods: According to body weight wistar rats were divided into five groups. Group I and Group II was treated with saline and capsaicin ( 3 mg/kg, i. v), while animals from Group III were treated with N( $\omega)$-nitro-L-arginine methyl ester (L-NAME) (30 mg/kg, i. v) $30 \mathrm{~min}$ before administration of capsaicin (3 mg/kg, i. v). Group IV animals were treated with glibenclamide $(10 \mathrm{mg} / \mathrm{kg}, \mathrm{i} . \mathrm{v}) 30 \mathrm{~min}$ before administration of capsaicin ( $3 \mathrm{mg} / \mathrm{kg}$, i. v). Group V was considered as a positive control and administered clopidogrel (30 mg/kg, p. o). Animals were subjected for in vitro, ex-vivo platelet aggregation assay. ADP $(30 \mu \mathrm{M})$ was utilized as an aggregating agent in these experiments. After these assays; animals of each group were subjected for subaqueous tail bleeding time in a rat model and $\mathrm{FeCl}_{3}$-induced arterial thrombosis model in rats.

Results: In ADP-induced in vitro platelet aggregation, a significant reduction in \% platelet aggregation was observed at $50 \mu \mathrm{M}(64.35 \pm 4.641)$ and $100 \mu \mathrm{M}(52.72 \pm 4.192)$ concentration of capsaicin as compared to vehicle control $(85.82 \pm 3.716)$. Capsaicin $(3 \mathrm{mg} / \mathrm{kg}$, i. v) also showed a significant reduction (49.53 \pm 4.075$)$ in ex-vivo ADP-induced platelet aggregation as compared to vehicle control (89.38 \pm 2.057$)$. In FeCl 3 induced arterial thrombosis model, Capsaicin ( $3 \mathrm{mg} / \mathrm{kg}$, i. v) exhibited an increase in time to occlusion in this rodent model and presence of the L-NAME and glibenclamide had inhibited the activity of capsaicin.

Conclusion: In our study, capsaicin $(50 \mu \mathrm{M}, 100 \mu \mathrm{M})$ exhibited potent anti-platelet activity in ADP-induced platelet aggregation, similarly capsaicin exhibited significant anti-platelet action in the ex-vivo study. Moreover, the presence of L-NAME and glibenclamide inhibited the anti-thrombotic and anti-platelet action of capsaicin. Therefore, it was concluded that $\mathrm{NO}$ and $\mathrm{K}_{\mathrm{ATP}}$ may be involved in the anti-thrombotic action of capsaicin.

Keywords: Capsaicin, Anti-platelet activity, Glibenclamide, Nitric Oxide, TRPV 1 channel

(C) 2018 The Authors. Published by Innovare Academic Sciences Pvt Ltd. This is an open access article under the CC BY license (http://creativecommons.org/licenses/by/4.0/) DOI: http://dx.doi.org/10.22159/ijpps.2018v10i4.22474

\section{INTRODUCTION}

Heart attacks and strokes are the most common causes of mortality and morbidity across the world and both represent clinical manifestations of acute arterial thrombosis [1]. Among them, atherosclerosis presents a greater risk to cardiovascular and peripheral vascular system causing mortality in cardiovascular disease patients [2]. Thrombus formation is a key mediator in the development of atherosclerosis [3]. The platelet is believed to play a pivotal role in pathogenesis and progression of atherosclerosis [4]. Previously it was believed that the platelet is having a minor role in this process; however, it is recognized that the platelet places itself as a critical link between thrombus formation, inflammation, and atherosclerosis [5]. At the site of vascular injury, platelets come into contact with subendothelial components and form a plug-like structure to avoid future damage to the endothelium. However, if the injury continues to happen, it will activate the cascade of signalling molecules which will form thrombus at the site of injury. This will lead to life-threatening disease states such as myocardial infarction, atherosclerosis or ischemic stroke [6].

Upon platelet formation at the site of vascular injury a series of cascade initiates which mainly involves three phases. Phase I, Phase II and Phase III name as the initial phase, extension phase and stabilization phase respectively. The initial phase involves attachment of platelets to the exposed sub-endothelial layer following vascular injury and a monolayer of activated cells is formed. This activated monolayer further recruits more additional platelets to construct another strong layer during extension phase
[7]. In order to restrict the recently formed thrombus to the initial injury site, regulation of platelet aggregation is the process to modulate a balance between activation and inhibition of signalling pathway of platelet [8]. Any defect in regulation of platelet activation or aggregation can cause arterial thrombosis, the major manifestation of atherosclerosis which triggers myocardial infarction and stroke. Nitric oxide (NO) is an endogenous gas present in endothelial cell of vascular endothelium, which is believed to play a predominant role in the regulation of platelet aggregation [9]. Endothelial NO responsible for maintenance of basal vascular tone and blood flow, and thereby regulation of blood pressure due to vasodilatory action [10].

A substantial amount of research has been carried out to elucidate the role of NO in platelet aggregation and found that NO can inhibit platelet activation or aggregation in vitro and in vivo [11]. The mechanism by which inhibition of platelet aggregation turns out by NO is caused by the cGMP-dependent pathway. Soluble guanylcyclase (sGC) is an enzyme responsible for the production of cyclic guanosine monophosphate (cGMP), and NO is involved in activation of SGC which further lead to activation of cGMPdependent protein kinase (PKG) via a cGMP-dependent pathway. This activated PKG caused inhibition of platelet activation via phosphorylating $\mathrm{T}_{\mathrm{x}} \mathrm{T} \mathrm{A}_{2}$ receptors and thus inhibits its aggregating action on platelet. Further details include inhibition of influx of $\mathrm{Ca}^{2+}$ and other positive ions via activating sarcoplasmic reticulum ATPase (SERCA) [12]. PKG is also involved in blockage of the release of $\mathrm{Ca}^{2+}$ cation from the sarcoplasmic reticulum via inhibition of 
inositol-1,4,5-trisphosphate, which also contributes to the lower level of intracellular cytosolic $\mathrm{Ca}^{2+}[13,14]$. Other than abovementioned mechanisms, two other mechanisms have been identified for platelet aggregation prevention caused by cGMP. It has been identified that cGMP inhibits phosphodiesterase type III enzyme and thereby causing elevation of the intracellular cAMP level; cGMP and cAMP act synergistically to inhibit platelet aggregation [15]. In another study, it has been identified that inhibition of $\mathrm{PI}_{3} \mathrm{~K}$ activation by cGMP leads to activation of $\mathrm{GP}_{\mathrm{II}} \mathrm{-}-\mathrm{II}_{\mathrm{a}}$ fibrinogen receptors [16]. Various cascades of pathways are being targeted in medical therapies to develop anti-platelet agents. These include therapies aimed at inhibiting $\mathrm{T}_{\mathrm{x}} \mathrm{A}_{2}, \mathrm{ADP}, \mathrm{GPII}_{\mathrm{b}} / \mathrm{III}_{\mathrm{a}}$, thrombin, collagen. Although major therapeutics is available in the area of thrombosis, disease prevalence is a cause of concern for healthcare industries in the world. Hence, we aimed to identify the role of capsaicin in platelet aggregation and mechanism of it. Capsaicin is a pungent photochemical present in peppers responsible for spiciness [17]. Capsaicin is a potent agonist of $\mathrm{TRPV}_{1}$ channel distributed throughout the body. TRPV1 channel plays a very important role in heat sensation [18]. Nitric oxide (NO) plays a pivotal role in pain sensation via activation of $\mathrm{TRPV}_{1}$ channel [19].

A number of few reports suggest that capsaicin have potential to inhibit platelet activation. Capsaicin was found to inhibit the aggregating effects of collagen in mouse platelet [20]. However, a significant amount of data spars regarding the detailed mechanism of action of capsaicin in platelet inhibitory action. To our best of knowledge, it has not been studied the role of capsaicin with respect to $\mathrm{NO}$ in platelet aggregation. Therefore, current investigation aimed to identify the role of capsaicin in platelet aggregation and elucidate the role of NO, $\mathrm{K}_{\mathrm{ATP}}$ in capsaicin mediated anti-platelet activity.

\section{MATERIALS AND METHODS}

\section{Animals}

Albino Wistar rats (150-200 g) were housed in a climate-controlled room (temperature $22 \pm 1{ }^{\circ} \mathrm{C}$; relative humidity $55 \% \pm 5 \%$ ) on a $12 \mathrm{~h}$ (light)-12 h (dark) cycle. Animals had access to a standard pellet diet (Certified Amrut brand rodent feed; Pranav Agro Industries, Pune, India) and filtered tap water ad libitum. All experiments were carried out with strict adherence to ethical guidelines and were conducted according to the protocol (LMCP/Cology/14/10) approved by the Institutional Animal Ethics Committee (IAEC), and according to Indian norms set by the Committee for the Purpose of Control and Supervision of Experiments on Animals (CPCSEA, New Delhi, India). In addition, the animals were cared for in accordance with the Guide to the Care and Use of Experimental Animals (Vol. 1, $2^{\text {nd }}$ ed., 1993, and Vol. 2, 1984) throughout the study. Throughout the entire study period, the animals were monitored for growth and health status to be certain that they were healthy.

\section{Chemicals}

Capsaicin, ADP (Adenosine Diphosphate) and Urethane were also purchased from Sigma Chemical, USA and ADP were prepared in phosphate buffer ( $\mathrm{pH}$ 7.4). $\mathrm{FeCl}_{3}$ was purchased from Hi-Media, India. Clopidogrel bisulfate was the generous gift from Zydus Cadila, India. In all experiments, water for injection was utilized for preparation of L-NAME and clopidogrel whereas capsaicin, glibenclamide was prepared in DMSO $(0.5 \%)+$ saline.

\section{Preparation of platelet-rich plasma (PRP) and platelet poor plasma (PPP)}

Blood was collected into the tubes containing 3.8\% trisodium citrate via retro-orbital route under light ether anesthesia from rats. All blood samples were centrifuged at $200 \mathrm{Xg}$ for $20 \mathrm{~min}$ and supernatant patelet rich plasma (PRP) was collected carefully. The remaining amount of blood was centrifuged at $800 \mathrm{Xg}$ for $20 \mathrm{~min}$ and supernatant platelet poor plasma (PPP) was collected with utmost care.

\section{Measurement of platelet aggregation using ADP as an aggregating agent}

Platelet aggregation studies were performed on microplate reader in 96-well, flat-bottomed, microtiter plates. A $180-\mu$ l volume of PRP was placed in each well, followed by addition of $20 \mu \mathrm{l}$ of ADP $(30 \mu \mathrm{M})$. For in vitro studies, PRP/PPP was incubated with various concentrations of capsaicin $(25,50$ and $100 \mu \mathrm{M})$ for $2 \mathrm{~min}$ at $37^{\circ} \mathrm{C}$ before addition of ADP. Readings were taken every 1-min over a 5min period at 405-nm wavelength. During the runtime, the plate was incubated at $37^{\circ} \mathrm{C}$ and was shaken vigorously in a shaking mode at the maximal speed available. All platelet aggregation studies were performed in triplicate. Change in optical density (OD) was measured by taking OD of buffer as blank.

$\%$ Aggregation was calculated using formula:

$\%$ Aggregation $=[($ Initial OD-Final OD $) /$ Initial OD $] * 100$

Experimental design for ex vivo platelet aggregation study in rats

On the basis of body weight wistar rats were randomly divided into following groups. Experimental design was given in fig. 1 .

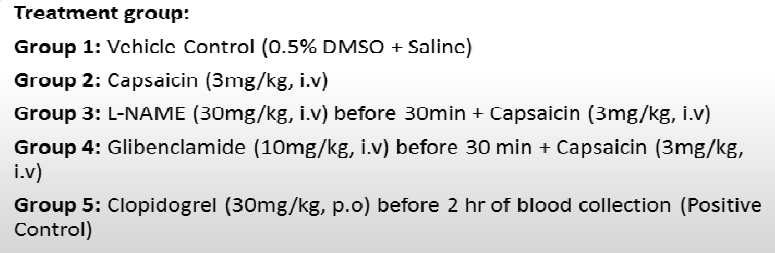

Fig. 1: Experimental design for ex vivo platelet aggregation study

Via the retro-orbital route blood samples were collected in tubes containing 3.8\% trisodium citrate under anesthesia. PRP/PPP was prepared as described above and samples were subjected to ADPinduced platelet aggregation assay. \% Aggregation was calculated in order to estimate the influence of capsaicin in platelet aggregation.

\section{Subaqueous tail bleeding time in rat}

Anaesthetized rats were fixed in supine position on a temperaturecontrolled $\left(37^{\circ} \mathrm{C}\right)$ heating table. After a defined latency period, the tail of the rat was transected with a razor-blade mounted on a selfconstructed device at a distance of $4 \mathrm{~mm}$ from the tip of the tail. Immediately after transection, the tail was immersed into a bath filled with isotonic saline solution $\left(37^{\circ} \mathrm{C}\right)$. The time until continuous blood flow ceased for $>30 \mathrm{~s}$ was measured, with a maximum observation time of $30 \mathrm{~min}$ (longer bleeding times were assigned a value of $30 \mathrm{~min})$. Clopidogrel $(30 \mathrm{mg} / \mathrm{kg}$, p. o., $120 \mathrm{~min}$ pretreatment time) was used as positive control.

\section{$\mathrm{FeCl}_{3}$-induced arterial thrombosis model in rats}

Rats $(n=6)$ were treated as per given protocol and then subjected to $\mathrm{FeCl}_{3}$-induced arterial thrombosis. The fecl 3 -induced chemical injury was used as a model of arterial thrombosis. A midline cervical incision was made on the ventral side of the neck and left carotid artery was isolated. Cannulation of the carotid artery was performed and connected to blood pressure measurement instrument (Biopac Systems, Inc, California, USA for B. P. measurement). A $2 \times 3 \mathrm{~mm}$ strip of Whatman filter paper No. \#1 saturated with $35 \%$ (w/v) $\mathrm{FeCl}_{3}$ was kept on the carotid artery for $5 \mathrm{~min}$. A sudden decrease in B. P. signalling was taken as an indicator of cessation of blood flow as a consequence to thrombus formation. Time to occlusion (TTO) was defined as the time from $\mathrm{FeCl}_{3}$ application to time of thrombus formation. A cutoff time was fixed at $60 \mathrm{~min}$ in case no thrombus formation was seen in drug-treated animals. Assessments of wet thrombus weight were also performed.

\section{Statistical analysis}

Results were expressed as mean \pm SEM. Data were analyzed by Oneway ANOVA followed by Dunnet's multiple comparison tests. All analysis was performed using graphpad prism software ${ }^{\circledR}$ version 6.0. $\mathrm{P}<0.05$ was considered to be statistically significant. 


\section{RESULTS}

Effects of capsaicin on ADP-induced in vitro and ex vivo platelet aggregation and mechanisms

In ADP-induced in vitro platelet aggregation, capsaicin exhibited a significant reduction in $\%$ platelet aggregation at $50 \mu \mathrm{M}$ $(64.35 \pm 4.641)$ and $100 \mu \mathrm{M}(52.72 \pm 4.192)$ concentration as compared to vehicle control (85.82 \pm 3.716$)$ (fig. 2). On another hand, capsaicin (3 mg/kg, i. v) also showed a significant reduction $(49.53 \pm 4.075)$ in ex-vivo ADP-induced platelet aggregation as compared to vehicle control $(89.38 \pm 2.057)$ (fig. 3). We also explored the mechanism of antiplatelet action of capsaicin by using L-NAME and glibenclamide to identify the role of NO and $\mathrm{K}_{\mathrm{ATP}}$ in capsaicin mediated antiplatelet action. Capsaicin at $3 \mathrm{mg} / \mathrm{kg}$, i. v exhibited a significant reduction in \% platelet aggregation $(49.53 \pm 4.075)$ as compared to vehicle control (89.38 \pm 2.057$)$ (fig. 3). Pretreatment of L-NAME somehow inhibited platelet inhibitory action of capsaicin, while on other hand addition of glibenclamide exhibited inhibition of anti-platelet actions of capsaicin shown in fig. 3 .

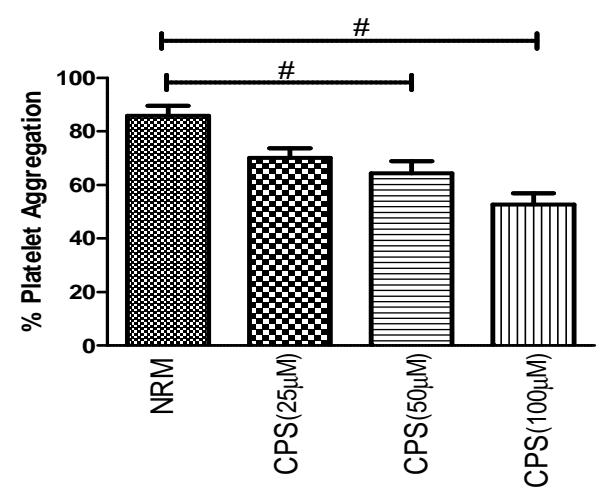

Fig. 2: Effects of capsaicin on ADP-induced platelet aggregation in vitro, NRM-normal control group, CPS-capsaicin treated group, \#=p<0.01 vs NRM

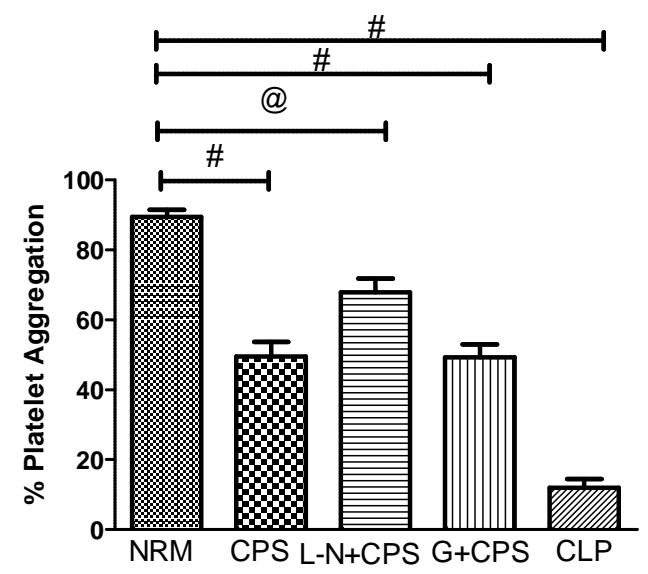

Fig. 3: Effects of capsaicin on ADP-induced platelet aggregation ex-vivo, NRM-normal control group, CPS= capsaicin-treated group ( $3 \mathrm{mg} / \mathrm{kg}$ ), L-N+CPS=30 mg/ $\mathrm{kg}$ L-NAME with $3 \mathrm{mg} / \mathrm{kg}$ Capsaicin, G+CPS=Glibenclamide $10 \mathrm{mg} / \mathrm{kg}$ with $3 \mathrm{mg} / \mathrm{kg}$ Capsaicin treated group, CLP=Clopidogrel $30 \mathrm{mg} / \mathrm{kg}$ treated group served as positive control; \#=p $<0.01$ vs NRM, @=p $<0.05$ vs NRM

Effects of capsaicin in vivo models of subaqueous tail bleeding time and $\mathrm{FeCl}_{3}$ induced thrombosis in rats and mechanisms involved

Following ex-vivo and in vitro platelet aggregation assay we carried out in vivo model of thrombosis to explore the role of capsaicin in thrombosis and its underlying mechanism. Capsaicin ( $3 \mathrm{mg} / \mathrm{kg}$, i. v.) $(446.3 \pm 38.75)$ showed a significant rise in bleeding time as compared to vehicle control $(241.8 \pm 17.94)$. Further, bleeding time for capsaicin $(3 \mathrm{mg} / \mathrm{kg}$, i. v.) is significantly lower than positive control clopidogrel (30 mg/kg, p. o.) (fig. 4). Bleeding time is a significant parameter of antiplatelet or anticoagulant activity; therefore it has a major impact on the identification of antiplatelet or anticoagulant effect of the substance. In our study, pretreatment of L-NAME significantly blocked the antiplatelet effect of capsaicin, while on another hand a significant rise in bleeding time was observed with the addition of glibenclamide to capsaicin as compared to vehicle control (fig. 4). However, bleeding time for glibenclamide pretreated group was significantly lower than positive control. Hence, it can be concluded that NO may be involved in the antiplatelet activity of capsaicin.

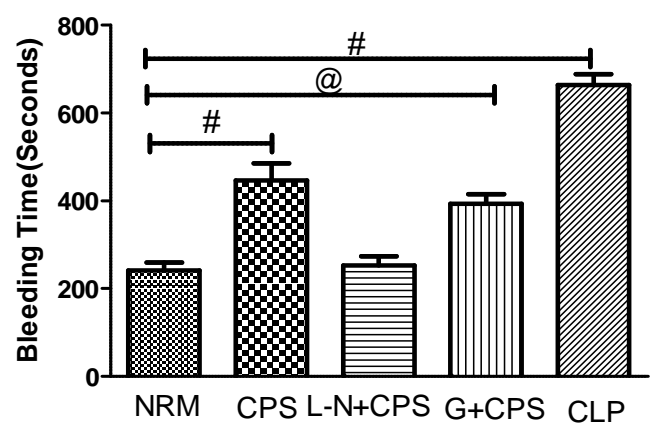

Fig. 4: Effects of capsaicin on bleeding time (Seconds). Inhibitors were used $30 \mathrm{~min}$ prior to capsaicin administration. NRM-Normal Control Group, CPS= Capsaicin treated Group (3 $\mathrm{mg} / \mathrm{kg}$ ), L-N+CPS=30 mg/kg L-NAME with $3 \mathrm{mg} / \mathrm{kg}$ Capsaicin, G+CPS=Glibenclamide $10 \mathrm{mg} / \mathrm{kg}$ with $3 \mathrm{mg} / \mathrm{kg}$ Capsaicin treated group, $\mathrm{CLP}=$ Clopidogrel $30 \mathrm{mg} / \mathrm{kg}$ treated group served as positive control; \#=p $<0.01$ vs NRM, @=p $<0.05$ vs NRM

Moreover, $\mathrm{FeCl}_{3}$ induced arterial thrombosis model was also performed to elucidate the role and mechanism of capsaicin in platelet aggregation. In that, time to occlusion (TTO) was measured as endpoint parameter. Capsaicin (3 mg/kg, i. v.) exhibited an increase in time to occlusion in this rodent model as shown in fig. below (fig. 5). This action was completely reversed in presence of L-NAME, while the presence of glibenclamide partially inhibited the activity of capsaicin as compared to vehicle control. Reduction in thrombus weight was observed in capsaicintreated group whereas L-NAME and glibenclamide exhibited inhibition of capsaicin activity partially depicted in the graph (fig. 6).

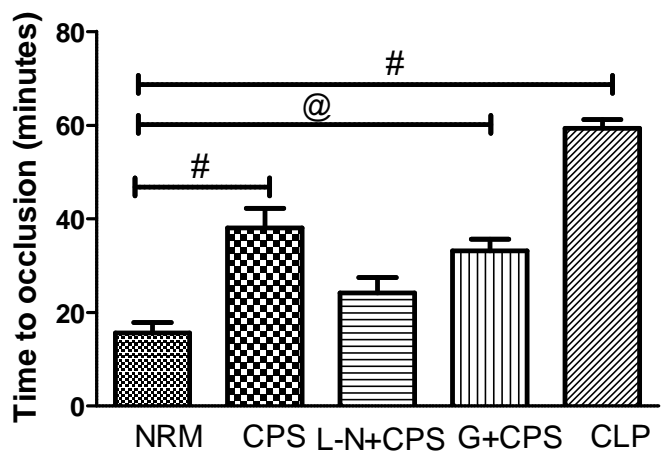

Fig. 5: Effects of capsaicin on time to carotid artery occlusion (TTO) using $\mathrm{FeCl} 3$-induced arterial thrombosis in male wistar rats. Inhibitors were used $\mathbf{3 0} \mathrm{min}$ prior to capsaicin administration. NRM-normal control group, CPS= capsaicin-treated group (3 $\mathrm{mg} / \mathrm{kg}$ ), L-N+CPS=30 mg/kg L-NAME with $3 \mathrm{mg} / \mathrm{kg}$ Capsaicin, G+CPS=Glibenclamide $10 \mathrm{mg} / \mathrm{kg}$ with $3 \mathrm{mg} / \mathrm{kg}$ Capsaicin treated group, $\mathrm{CLP}=\mathrm{Clopidogrel} 30 \mathrm{mg} / \mathrm{kg}$ treated group served as positive control; \#=p<0.01 vs NRM, @=p $<0.05$ vs NRM 


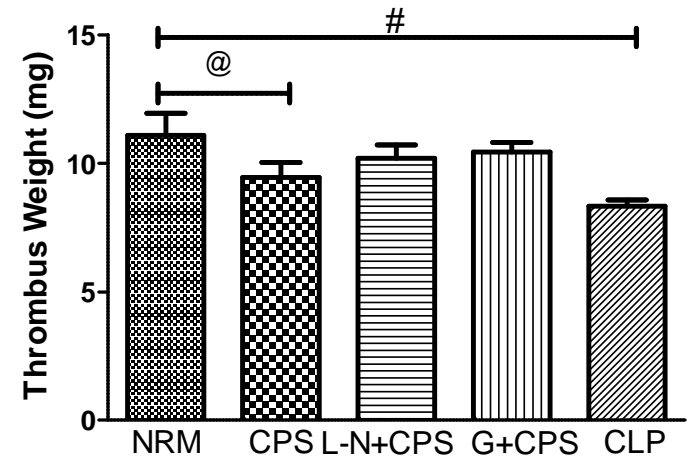

Fig. 6: Effects of capsaicin on thrombus wet weight FeCl3induced thrombosis model in rats. NRM-normal control group, CPS = capsaicin-treated group $(3 \mathrm{mg} / \mathrm{kg}), \mathrm{L}-\mathrm{N}+\mathrm{CPS}=30 \mathrm{mg} / \mathrm{kg} \mathrm{L}-$ NAME with $3 \mathrm{mg} / \mathrm{kg}$ Capsaicin, G+CPS=Glibenclamide $10 \mathrm{mg} / \mathrm{kg}$ with $30 \mathrm{mg} / \mathrm{kg}$ Capsaicin treated group, CLP=Clopidogrel 30 $\mathrm{mg} / \mathrm{kg}$ treated group served as positive control; \#=p<0.01 vs NRM, @=p $<0.05$ vs NRM

\section{DISCUSSION}

Antiplatelet activity of capsaicin has been studied in many literatures; however, mechanism of its action remains a controversial. Here, we tried to identify the role of various signaling molecules in antiplatelet activity of capsaicin. It is well-known fact that NO plays an important role in modulation of platelet activity. Now days, platelet aggregation is a prime topic of discussion because of dependency of leading cardiovascular diseases such as myocardial infarction and atherosclerosis on platelet modulation. Transient receptor potential vanilloid receptor $1\left(\mathrm{TRPV}_{1}\right)$, has established its pivotal role in the modulation of pain and inflammatory responses in neurons via in multiple chemical and physical stimuli [21]. It is well known fact that capsaicin serves as a potent agonist of $\mathrm{TRPV}_{1}$ and activates these channels [22]. The activation of $\mathrm{TRPV}_{1}$ channels results in increased $\left[\mathrm{Ca}^{2+}\right]_{i}$ level and thereby downstream of signaling cascades activation takes place. Besides its pivotal role in pain modulation, the $\mathrm{TRPV}_{1}$ channels are also found to be playing a major role in regulating the physiological functions of cardiovascular cells and the pathogenesis of cardiovascular diseases [23]. In spite of progress in research of capsaicin and cardiovascular research in last decade, the role and effects of capsaicin on platelet aggregation are not studied well. Several reports states that activation of TRPV 1 channels in endothelial cells protects against atherosclerosis, hypertension and stroke via activation of NO [24]. However, role of NO is not elucidated well in platelet function. Hence, we correlated capsaicin with $\mathrm{TRPV}_{1}$ to determine the role of NO using platelet aggregation assay and arterial model of thrombosis.

There are limited reports available suggesting antithrombotic effects of capsaicin and direct role of capsaicin in in vivo thrombosis model has not been studied well. Therefore, we investigated the role of capsaicin in antiplatelet activity and evaluated its mechanism(s). We identified that capsaicin exhibited significant anti-platelet effect at $100 \mu \mathrm{M}$ concentration as compared to vehicle control in in vitro platelet aggregation study. Moreover, we investigated the role of NO and $\mathrm{K}_{\mathrm{ATP}}$ by using L-NAME and glibenclamide, respectively in ex vivo and in vivo model of thrombosis. It was observed that capsaicin (3 $\mathrm{mg} / \mathrm{kg}$, i. v.) significantly reduced platelet aggregation in in vitro and ex-vivo study and pretreatment of L-NAME and glibenclamide partially blocked anti-platelet effect of capsaicin in similar models suggests NO and $\mathrm{K}_{\text {ATP }}$ may have a role in the anti-platelet effect of capsaicin. Going further, we investigated the role of capsaicin in an arterial model of thrombosis and found that capsaicin significantly increased the time to occlude in $\mathrm{FeCl}_{3}$ induced thrombus formation model as compared to vehicle control and interestingly, pretreatment of L-NAME and KATP blocked the antithrombotic action of capsaicin in the same model. Above observations suggest that capsaicin may have antithrombotic activity via NO-cGMP pathway. A drug characteristic belongs to antiplatelet or anticoagulant can be identified on the basis of bleeding profile of the drug, hence we studied the bleeding profile of capsaicin using tail vein bleeding time rodent model. We observed that bleeding time for the capsaicintreated group was significantly decreased as compared to vehicle control but much more than positive control (clopidogrel). Therefore, it can be concluded that capsaicin has a little effect on blood coagulation but not significant. It has been demonstrated that thrombus composition is an indicator of coronary occlusion time. "Fresh" thrombi have the highest portion of platelets, while the proportion of fibrin fibers increases over time, as the level of thrombin increases, leading to "older" fibrin-rich thrombi [25]. In our study, thrombus formed by $\mathrm{FeCl}_{3}$ was less than within a day hence it is to be considered as fresh thrombus composed of high portion of platelet and it is also documented in previous studies. In a model of $\mathrm{FeCl}_{3}$ induced thrombus, capsaicin has significantly showed inhibitory action on thrombus formation. Therefore, it can be concluded that capsaicin may exert antithrombotic effect by inhibiting structural platelet inhibition via induction of NO from endothelium. In our result, effect of fibrin was not observed due to thrombin structure was confined to rich level of platelet than fibrin clot. Hence, our study supports the previous finding of antiplatelet activity of capsaicin [26]. Eguchi et al. demonstrated that endothelial cell mitochondria have decisive role on thrombus formation process. Upon injury to endothelium, reactive oxygen species (ROS) enhance the process of thrombus formation which is attenuated by $\mathrm{K}_{\mathrm{ATP}}$ channel opener [27]. In our study of $\mathrm{FeCl}_{3}$ induced thrombosis; thrombus was formed as a result of damage to the endothelium was caused by $\mathrm{FeCl}_{3}$. This thrombus formation time and its extent were attenuated by the treatment of capsaicin. However, addition of glibenclamide to capsaicin somehow shifted the antithrombotic activity of capsaicin towards $\mathrm{K}_{\mathrm{ATP}}$ channel which was not significant. Our results are not consistent with observation of Mittelstadt who stated that Capsaicininduced inhibition of platelet aggregation is not mediated by $\mathrm{TRPV}_{1}$ [28]. In our experiments results, partial involvement of $K_{\text {ATP }}$ was seen in various parameters of arterial model of thrombosis hence the role of $\mathrm{K}_{\mathrm{ATP}}$ channel in capsaicin offered antithrombotic action could not be neglected. Therefore, it would not be wrong to say that activation of $\mathrm{TRPV}_{1}$ channel by capsaicin may have opened $\mathrm{K}_{\mathrm{ATP}}$ in the endothelium of artery of rat.

\section{CONCLUSION}

In conclusion, our current investigation also supports previous finding showing capsaicin's role in platelet aggregation, moreover, we have also investigated the mechanism of antiplatelet activity of capsaicin. We have found that NO plays a predominant role in the anti-platelet action of capsaicin via activation of sGC-cGMP modulatory signalling pathway and shift of anti-thrombotic activity towards $\mathrm{K}_{\text {ATP }}$ channel was also evident from our current investigation result. This may be due to activation of $\mathrm{TRV}_{1}$ by capsaicin. One previous report stated antiplatelet activity of capsaicin is unrelated to $\mathrm{TRPV}_{1}$, while in our investigation it has been showed that role of $\mathrm{TRPV}_{1}$ in thrombosis of artery via $\mathrm{K}_{\mathrm{ATP}}$ cannot be neglected.

\section{ACKNOWLEDGEMENT}

The authors are grateful to the Department of Pharmacology, L. M. College of Pharmacy, Ahmedabad and C. U. Shah University, Wadhwan city.

\section{AUTHORS CONTRIBUTIONS}

Mihir Patel carried out the experimental part of the work, performed data analysis and drafted and revised the manuscript. The design of the work and correction of the manuscript was done by the corresponding author Dr. Anita Mehta and Kiranj Chaudagar. All authors read and approved the final manuscript.

\section{CONFLICT OF INTERESTS}

The authors declare that there are no conflicts of interest associated with this study.

\section{REFERENCES}

1. Mozaffarian D, Benjamin EJ, Go AS, Arnett DK, Blaha MJ, Cushman $\mathrm{M}$, et al. American heart association statistics 
committee.; stroke statistics subcommittee. Heart disease and stroke statistics-2016 update: a report from the American heart association. Circulation 2016;133:338-60.

2. National Institutes of Health: National Heart, Lung and Blood Institute. NHLBI Morbidity and Mortality Chart Book. National Heart, Lung and Blood Institute; 2009.

3. Libby P, Ridker PM, Hansson GK. Leducq transatlantic network on atherothrombosis. Inflammation in atherosclerosis: from pathophysiology to practice. J Am Coll Cardiol 2009;54:2129-38.

4. Davi G, Patrono C. Platelet activation and atherothrombosis. N Engl J Med 2007;357:2482-94.

5. Furie B, Furie BC. Mechanisms of thrombus formation. N Engl J Med 2008;359:938-49.

6. Cosemans JM, Munnix IC, Wetzker R, Heller R, Jackson SP, Heemskerk JW. Continuous signalling via PI3K isoforms beta and gamma is required for platelet ADP receptor function in dynamic thrombus stabilization. Blood 2006;108:3045-52.

7. Jackson SP, Nesbitt WS, Kulkarni S. Signaling events underlying thrombus formation. J Thromb Haemost 2003;1:1602-12.

8. Gibbins JM. Platelet adhesion signalling and the regulation of thrombus formation. J Cell Sci 2004;117:3415-25.

9. Heusch G, Post H, Michel MC, Kelm M, Schulz R. Endogenous nitric oxide and myocardial adaptation to ischemia. Circ Res 2000;87:146-52.

10. Gewaltig MT, Kojda G. Vasoprotection by nitric oxide: mechanisms and therapeutic potential. Cardiovasc Res 2002; 55:250-60.

11. Radomski MW, Palmer RM, Moncada S. The anti-aggregating properties of vascular endothelium: interactions between prostacyclin and nitric oxide. Br J Pharmacol 1987;92:639-46.

12. Trepakova ES, Cohen RA, Bolotina VM. Nitric oxide inhibits capacitative action influx in human platelets by promoting sarcoplasmic/endoplasmic reticulum $\mathrm{Ca}^{2+}$-ATPase-dependent refilling of $\mathrm{Ca}^{2+}$ stores. Circ Res 1999;84:201-9.

13. Wang GR, Zhu Y, Halushka PV, Lincoln TM, Mendelsohn ME. Mechanism of platelet inhibition by nitric oxide: in vivo phosphorylation of thromboxane receptor by cyclic GMPdependent protein kinase. Proc Natl Acad Sci USA 1998;95:4888-93.

14. Schlossmann J, Ammendola A, Ashman K, Zong X, Huber A, Neubauer $\mathrm{G}$, et al. Regulation of intracellular calcium by a signalling complex of IRAG, IP3 receptor and cGMP kinase Ibeta. Nature 2000;404:197-201.

15. Maurice DH, Haslam RJ. Molecular basis of the synergistic inhibition of platelet function by nitrovasodilators and activators of adenylate cyclase: inhibition of cyclic AMP breakdown by cyclic GMP. Mol Pharmacol 1990;37:671-81.

16. Radomski MW, Palmer RM, Moncada S. Endogenous nitric oxide inhibits human platelet adhesion to vascular endothelium. Lancet 1987;2:1057-8.

17. Kosuge $S$, Inagaki $Y$, Okumura $H$. Studies on the pungent principles of red pepper. Part 11. Determination and contents of the two pungent principles. J Agric Chem Soc 1962;36:251.

18. Caterina MJ, Schumacher MA, Tominaga M, Rosen TA, Levine JD, Julius D. The capsaicin receptor: a heat-activated ion channel in the pain pathway. Nature 1997;389:816-24.

19. Meller ST, Dykstra C, Gebhart GF. Production of endogenous nitric oxide and activation of soluble guanylate cyclase are required for $\mathrm{N}$-methyl-D-aspartate-produced facilitation of the nociceptive tail-flick reflex. Eur J Pharmacol 1992;214:93-6.

20. Wang JP, Hsu MF, Teng CM. Antiplatelet effect of capsaicin. Thromb Res 1984;36:497-507.

21. Caterina MJ, Rosen TA, Tominaga M, Brake AJ, Julius D. A capsaicin-receptor homologue with a high threshold for noxious heat. Nature 1999;398:436-41.

22. Venkatachalam K, Montell C. TRP channels. Annu Rev Biochem 2007;76:387-17.

23. Yang D, Luo Z, Ma S, Wong WT, Ma L, Zhong J, et al. Activation of TRPV1 by dietary capsaicin improves endotheliumdependent vasorelaxation and prevents hypertension. Cell Metab 2010;12:130-41.

24. Ching LC, Kou YR, Shyue SK, Su KH, Wei J, Cheng LC, et al. Molecular mechanisms of activation of endothelial nitric oxide synthase mediated by transient receptor potential vanilloid type 1. Cardiovasc Res 2011;91:492-501.

25. Shand RA, Butler KD, Davies JA, Menys VC, Wallis RB. The kinetics of platelet and fibrin deposition on to damaged rabbit carotid arteries in vivo: involvement of platelets in the initial deposition of fibrin. Thromb Res 1987;45:505-15.

26. Wang JP, Hsu MF, Hsu TP, Teng CM. Antihemostaticand antithrombotic effects of capsaicin in comparison with aspirin and indomethacin. Thromb Res 1985;37:669-79.

27. Eguchi $Y$, Takahari $Y$, Higashijima N, Ishizuka N, Tamura N, Kawamura Y, et al. Nicorandil attenuates FeCl-induced thrombus formation through the inhibition of reactive oxygen species production. Circ J 2009;73:554-61.

28. Mittelstadt SW, Nelson RA, Daanen JF, King AJ, Kort ME, Kym $\mathrm{PR}$, et al. Capsaicin-induced inhibition of platelet aggregation is not mediated by transient receptor potential vanilloid type 1 . Blood Coagul Fibrinolysis 2012;23:94-7. 